\title{
Cardiovascular changes in patients with adult-onset growth hormone deficiency assessed by CMR
}

\author{
Abhishek Dattani $i^{*}$, Julia Thomas², Filip Zemrak1, Thomas R Burchell ${ }^{1}$, Steffen E Petersen ${ }^{1}$, Ashley Grossman³ \\ Marta Korbonits ${ }^{2}$, Ceri Davies ${ }^{1}$ \\ From 15th Annual SCMR Scientific Sessions \\ Orlando, FL, USA. 2-5 February 2012
}

\section{Summary}

The study investigated cardiovascular changes in adultonset growth hormone deficiency (GHD) and showed that patients with adult-onset GHD have a left ventricular mass index (LVMi) at or below the lower limit of normal, which improves with one year of growth hormone replacement.

\section{Background}

GHD causes cardiovascular problems, with loss of cardiac response to exercise and increased cardiac mortality, however the underlying processes are poorly understood.

\section{Methods}

Ten patients with adult-onset GHD and age- and sexmatched controls $(n=10)$ underwent CMR. Patients underwent scans before disease treatment and at twelve months after treatment. Cardiac parameters were calculated and indexed to body surface area (BSA). The comparison between groups was done using Mann-UWhitney test and within the group using Wilcoxon test. The data are presented as median values.

\section{Results}

Patients with GHD did not have significantly different left ventricle (LV) mass or volumetric parameters from controls: LV mass index (LVMi) 55.0 vs. $56.6 \mathrm{~g} / \mathrm{m}^{2}$, $\mathrm{p}=0.315$; end diastolic volume index (EDVi) 69.2 vs. 76.3 $\mathrm{ml} / \mathrm{m}^{2}, \mathrm{p}=0.1655$; end systolic volume index (ESVi) 24.8 vs. $28.3 \mathrm{ml} / \mathrm{m}^{2}, \mathrm{p}=0.3527$ and ejection fraction (EF) 64 vs. $63 \%, p=0.8197$. However, patients had an LVMi beneath the lower limit of normal when compared to published normal ranges $\left(55.4-74.0 \mathrm{~g} / \mathrm{m}^{2}\right)$.

${ }^{1}$ Cardiovascular Biomedical Research Unit, Barts and the London School of Medicine and Dentistry, Queen Mary, University of London, London, UK Full list of author information is available at the end of the article
There were no differences between right ventricular (RV) EDVi (68.0 vs. $\left.80.8 \mathrm{ml} / \mathrm{m}^{2}, \mathrm{p}=0.393\right)$, ESVi (26.2 vs. $27.5, \mathrm{p}=0.7394)$ and $E F(63$ vs. $61 \%, \mathrm{p}=0.9696)$ in the GHD group and controls.

Patients with GHD on GH treatment for 1 year showed an increase in median insulin-like growth factor I (IGF-I) SDS from -1.83 to $+0.40(\mathrm{p}=0.0068)$. There was no correlation between LVMi and IGF-I SDS $(r=0.164, p=0.657)$. At one year the median LVMi moved into the previously published normal range (55.0 to $63.0 \mathrm{~g} / \mathrm{m}^{2}$, normal range 55.4 - 74.0) achieving statistical significance compared to pre-treatment values $(\mathrm{p}=0.0156)$.

\section{Conclusions}

Patients with adult-onset GHD have an LVMi at or below the lower limit of normal, which improves with one year of growth hormone replacement.

\section{Funding}

Supported by departmental grants by Pfizer and Novartis.

\section{Author details}

${ }^{1}$ Cardiovascular Biomedical Research Unit, Barts and the London School of Medicine and Dentistry, Queen Mary, University of London, London, UK. ${ }^{2}$ Centre for Endocrinology, William Harvey Research Institute, Barts and the London School of Medicine and Dentistry, Queen Mary, University of London, London, UK. ${ }^{3}$ Oxford Centre for Diabetes, Endocrinology and Metabolism, University of Oxford, Oxford, UK.

Published: 1 February 2012

doi:10.1186/1532-429X-14-S1-P192

Cite this article as: Dattani et al:: Cardiovascular changes in patients with adult-onset growth hormone deficiency assessed by CMR. Journal of Cardiovascular Magnetic Resonance 2012 14(Suppl 1):P192.

\section{C)

(C) 2012 Dattani et al; licensee BioMed Central Ltd. This is an open access article distributed under the terms of the Creative Commons Attribution License (http://creativecommons.org/licenses/by/2.0), which permits unrestricted use, distribution, and reproduction in any medium, provided the original work is properly cited. 